\title{
USO DE MEZCLADORES ESTÁTICOS PARA MEJORAR LAS CURVAS DE CALIBRACIÓN EN EL ANÁLISIS DE GASES DE IMPORTANCIA BIOLÓGICA
}

\author{
USE OF STATIC MIXERS FOR ENHANCING STANDARD CURVES IN THE ANALYSIS OF \\ BIOLOGICALLY IMPORTANT GASES
}

\author{
Alejandro Martínez-Flores, Teodoro Espinosa-Solares, J. Joel Corrales-García ${ }^{\star}$ y J. Guillermo Cruz-Castillo
}

Posgrado de Ciencia y Tecnología Agroalimentaria, Universidad Autónoma Chapingo. Km 38.5 Carretera México-Texcoco. 56230, Chapingo, Estado de México. México. Tel. 01 (595) 2-1500 ext. 5177.

${ }^{*}$ Autor para correspondencia (joelcorrales@hotmail.com)

\section{RESUMEN}

La evaluación de la evolución de gases de importancia biológica para estudios de fisiología poscosecha de productos hortícolas, así como la elaboración y el uso de curvas de calibración para este tipo de trabajos, son tareas necesarias. Sin embargo, el mezclado de gases para la elaboración de curvas de calibración no siempre es altamente eficiente. Generalmente, el mezclado de gases se lleva a cabo en un recipiente común para gases y se supone que los gases se mezclarán de inmediato solo con ponerlos en contacto, lo cual no es necesariamente cierto. El objetivo de este estudio fue evaluar el desempeño de un sistema de mezcladores estáticos en la elaboración de curvas de calibración confiables. Para cada punto de referencia de las curvas de calibración se prepararon diferentes diluciones de etileno con nitrógeno, con y sin el uso de un sistema de mezcladores estáticos. Se usó un cromatógrafo de gases para evaluar la concentración de las mezclas gaseosas. Cuando se empleó el sistema de mezcladores estáticos para la preparación de la mezcla de gases, se obtuvo una curva más confiable y una reducción significativa (hasta de $87 \%$ ) del error estándar en la estimación de los valores de referencia promedio en cada punto de dilución. El uso de un sistema de mezcladores estáticos permite elaborar curvas de calibración más confiables, en comparación con hacerlas sin el uso de este sistema.

Palabras clave: Mezclado de gases, análisis de gases, curvas tipo, calibración, medición de etileno, medición de tasas de respiración.

\section{SUMMARY}

Assessing the evolution of biological important gasses for postharvest studies of horticultural crops, as well as, elaborating and using calibration curves for this type of studies, are necessary tasks. Nevertheless, gas mixing to determine calibration curves is not always highly efficient. Usually, gas mixing takes place in a common gas container, assuming that gasses will mixed immediately. However, this is not necessarily true. The objective of this study was to evaluate the performance of a static mixers system on the elaboration of reliable calibration curves. Different dilutions of ethylene with nitrogen were prepared with and without using a static mixers system, for each reference point. Gas chromatography was used to evaluate gas concentrations. When the static mixers system was used for preparing the mix, a more reliable calibration curve was obtained and a significant reduction in the standard error (up to $87 \%$ ) was achieved. Thus, using a static mixers system allows more reliable calibration curves.

Index words: Gas mixing, gas analysis, type curves, calibration, ethylene measurement, respiration rates measurement.

\section{INTRODUCCIÓN}

El estudio de la fisiología poscosecha incluye, entre otras cosas, la producción, intercambio y acumulación de gases de importancia biológica, como oxígeno $\left(\mathrm{O}_{2}\right)$, bióxido de carbono $\left(\mathrm{CO}_{2}\right)$, y etileno $\left(\mathrm{C}_{2} \mathrm{H}_{4}\right)$, entre otros. Los dos primeros son indicadores de la respiración del producto, y el tercero es la hormona que promueve maduración y senescencia (Kader, 2007a). En el ámbito de la fisiología y tecnología postcosecha es fundamental determinar la cantidad producida, intercambiada o acumulada de estos gases, dado que la evolución de sus concentraciones es un indicador de la actividad metabólica y permite determinar con precisión la manifestación de síntomas de algunas fisiopatías, así como 
respuestas fisiológicas que presentan los productos a determinadas condiciones de manejo (temperatura, humedad relativa, entre otras), para mantener las propiedades del fruto u hortaliza, acelerar su maduración, o para determinar su potencial de vida de anaquel y el calor vital de los productos; esto último es necesario para el diseño o la adopción de sistemas de enfriamiento y de frigoconservación. Con esta información es posible determinar el efecto que tiene la tecnología en la conservación poscosecha de productos hortícolas, así como verificar si existen alteraciones metabólicas en dichos productos.

La concentración de los gases se determina comúnmente por cromatografía de gases (CG), para lo cual es necesario contar con gases de referencia de varias concentraciones conocidas (estándares), que por lo regular son costosos, o construir curvas de calibración a partir de concentraciones conocidas del gas de interés. Lo ideal es construir curvas confiables con muchos puntos de referencia, mediante diluciones a partir de un sólo estándar de concentración alta, conocida y certificada del gas de interés, y mezclarlo adecuadamente con un gas inerte (Kader, 2007b). Sin embargo, el mezclado de gases para hacer las diluciones, y las curvas resultantes, no siempre resulta eficiente, porque los datos muestran alta variabilidad y generan incertidumbre en el resultado generado, lo que obliga a incrementar el número de repeticiones con el consecuente aumento en costos y en tiempo de elaboración.

El mezclado de gases está basado en relaciones de sus masas, volúmenes o presiones. En estudios postcosecha, para la mezcla de gases se utilizan sistemas estáticos o dinámicos. En los dinámicos (mezclado en flujo continuo) los gases se mezclan en función de su volumen a presión y temperatura constante, mediante dispositivos que controlan el flujo, como válvulas y tubos capilares (Kader, 2007b). Este método implica conducir los flujos de los gases hacia un recipiente donde se mezclan, lo que conduce eventualmente, pero no necesariamente de forma inmediata, a la dilución deseada del gas de interés. Lógicamente, la eficiencia del mezclado afecta los resultados (valor promedio de cada punto de referencia). Si la eficiencia es alta, entonces los valores promedio se originan de valores individuales poco dispersos (con mínima varianza) y confiables. Es de suponer que si el mezclado de gases fuera eficiente, se obtendrían mezclas más homogéneas de manera inmediata, con valores de referencia promedio menos dispersos y con los cuales se podrían elaborar curvas de calibración precisas y confiables. En relación con lo anterior, algunos autores (Barrué et al., 2001; Couvert et al., 2006; Patkar y Patwardhan, 2011) han estudiado los factores que influyen en la eficiencia del mezclado de sistemas gas-gas, lo que evidencia que se trata de un tema en proceso de investigación.

Los mezcladores estáticos consisten de varios elementos idénticos de mezclado sin movimiento colocados a lo largo de un tubo o canal, generalmente rotados $90^{\circ}$ con respecto al elemento anterior; este cambio periódico en la geometría de los mezcladores estáticos produce reorientación y redistribución de los fluidos. La creación de flujos secundarios o reorientación permite mezclar diferentes corrientes de fluidos que fluyen a través del tubo. Estos dispositivos han tenido diversas aplicaciones, como el mezclado de fluidos altamente viscosos (Fourcade et al., 2001), dispersión de gas en líquido (Fradette et al., 2006) y mezclado de gases (Couvert et al., 2006). Uno de los mezcladores estáticos de mayor aplicación ha sido el Kenics@ (Chemineer,Inc.) (Tajima et al. 2010). Con base en el buen desempeño que tienen estos mezcladores, el objetivo del presente trabajo fue evaluar curvas de calibración para etileno, mediante CG a partir de mezclas gaseosas de etileno con nitrógeno como gas diluyente, con y sin el empleo de un sistema de mezcladores estáticos, para así extender su aplicación en la fisiología y tecnología postcosecha.

\section{MATERIALES Y MÉTODOS}

Se construyeron curvas de calibración de etileno a partir de un estándar de etileno (100 ppm balance $\mathrm{N}_{2}$; Praxair de México, S.A., México). Para diluir el gas estándar se utilizó nitrógeno (grado certificado alta pureza: 99.9 \%; Praxair de México, S.A., México). El flujo $\left(\mathrm{L} \mathrm{h}^{-1}\right)$ de ambos gases fue controlado con ayuda de dos tableros (uno para cada gas), como los utilizados para medir respiración de frutos por el método de Claypool y Keefer modificado por Pratt y Mendoza (1979). Los tableros permiten controlar de manera práctica, inmediata y confiable el flujo de los gases que se mezclan (Figura 1). La altura de las columnas de agua del tablero es un indicador de la presión de trabajo del sistema, pero también de la velocidad de flujo del gas. 


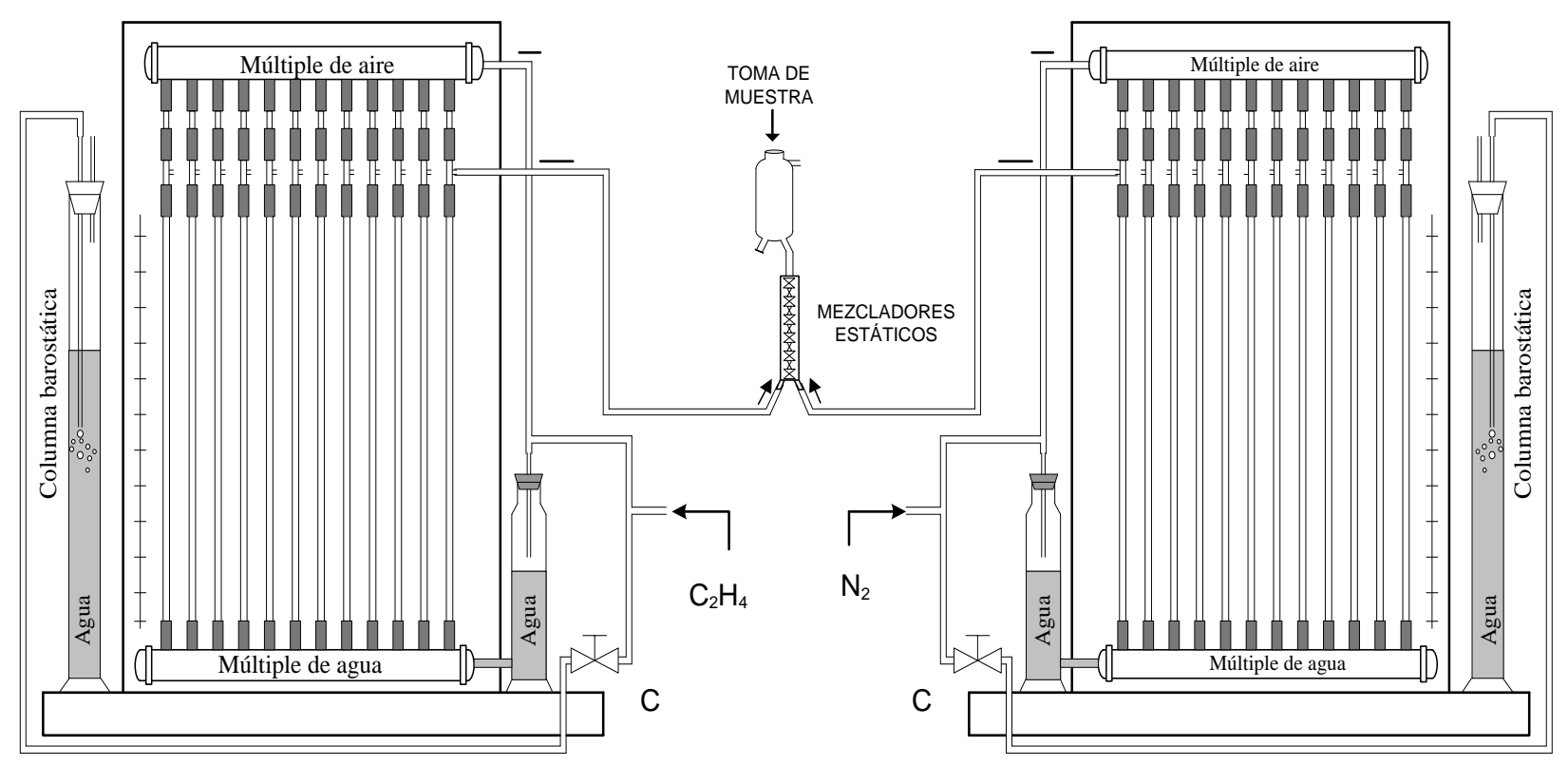

Figura 1. Representación esquemática del sistema utilizado para el control del flujo y mezclado de gases para la obtención más eficiente de mezclas diluidas de etileno con nitrógeno.

Entonces, para obtener cada punto de referencia de la curva, de acuerdo con el flujo requerido de gas para ese punto de referencia, los gases se suministraron al tablero cuyo sistema de conexiones mantiene el nivel de agua de los tubos verticales hasta la altura de columna de agua (presión) correspondiente al flujo requerido de cada gas para ese punto de referencia de la curva en particular; estos flujos fueron previamente determinados. Ambos gases se mezclaron en diferentes proporciones, para generar a voluntad diferentes mezclas diluidas del gas de interés, de modo que conforme se disminuía el flujo del gas a diluir (etileno), el flujo del gas diluyente $\left(\mathrm{N}_{2}\right)$ aumentaba en la misma proporción. La toma de muestras se hizo después de haber pasado por mezcladores estáticos.

Los flujos se midieron para cada gas a diferentes presiones (a diferentes alturas de columna de agua). Esta medición se obtuvo del promedio de tres repeticiones y se llevó a cabo con un medidor de flujo de vidrio (Varian ${ }^{\circledR}$ mod. 03-949846-00) y un cronómetro.

Los dispositivos estáticos empleados para el mezclado de gases, de la marca Kenics $\odot$ (Chemineer, Inc.) (Figura 2), están conformados por secciones metálicas de corte helicoidal, con cambios de la dirección de flujo a $90^{\circ}$ cuando se cambia de una sección a otra.

Para la preparación de las mezclas se eligió un flujo máximo de etileno $\left(11 \mathrm{~L} \mathrm{~h}^{-1}\right)$, éste se fue diluyendo progresivamente con el flujo de nitrógeno de alta pureza, considerando que la suma de ambos flujos fuera el máximo de la inicial del etileno $\left(11 \mathrm{~L} \mathrm{~h}^{-1}\right)$.

\section{Tratamientos}

Se elaboraron curvas de calibración bajo dos condiciones (tratamientos) y con tres repeticiones. El primer tratamiento consistió en elaborar la curva de calibración tal como se hace normalmente, es decir, simplemente poniendo en contacto los flujos de ambos gases en un dispositivo de vidrio sin mezclador, de cuya salida se extrajeron las muestras gaseosas para su análisis en el cromatógrafo. En el segundo tratamiento los flujos de ambos gases se hicieron pasar a través de dos mezcladores estáticos colocados dentro de tubos de vidrio y conectados en serie, de cuya salida se extrajeron las muestras gaseosas para análisis. 


\section{Variables medidas}

En ambos tratamientos se extrajeron muestras de 100 $\mu \mathrm{L}$ que se inyectaron a un cromatógrafo de gases marca Varian ${ }^{\circledR}$ mod. 3400 con el software Star Chromatography Workstation Versión 4.5 (Varian, 1996). En el cromatógrafo se empleó una columna capilar Chrompack ${ }^{\circledR}$ de $25 \mathrm{~m}$ de longitud, $0.32 \mathrm{~mm}$ de diámetro interno y $0.45 \mathrm{~mm}$ de diámetro externo. La presión en la cabeza de la columna fue de 199.948 a $206.843 \mathrm{kPa}(29$ a $30 \mathrm{psi})$. Las condiciones de temperatura fueron: $80^{\circ} \mathrm{C}$ en la columna, $150{ }^{\circ} \mathrm{C}$ en el inyector, $150{ }^{\circ} \mathrm{C}$ en el auxiliar, $170{ }^{\circ} \mathrm{C}$ en el detector de ionización de flama (FID) y 200 ${ }^{\circ} \mathrm{C}$ en el filamento. El tiempo de corrida fue de $4 \mathrm{~min}$. En estas condiciones el tiempo de retención del etileno fue de $3.063 \mathrm{~min}$.

La concentración teórica de etileno para cada dilución se calculó con base en un balance de masa del etileno en el proceso de mezclado (Ecuación 1).

$\left[\right.$ Etileno $\left._{\text {mezcla }}\right]=\frac{F_{\text {etileno }}\left[\text { Etileno }_{\text {estándar }}\right]}{F_{\text {mezcla }}}$

Donde:

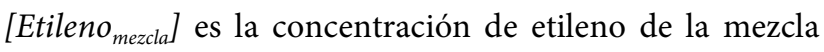
[ $\left.\mathrm{mg} \mathrm{L}^{-1}\right]$; [Etileno estandar $_{\text {] }}$ es la concentración de etileno gas estándar $\left[\mathrm{mg} \mathrm{L}^{-1}\right] ; F_{\text {mezcla }}$ es el flujo volumétrico (total) de la mezcla $\left(\mathrm{L} \mathrm{h}^{-1}\right) ; y F_{\text {etileno }}$ es el flujo volumétrico de etileno (gas estándar) $\left(\mathrm{L} \mathrm{h}^{-1}\right)$.

En el Cuadro 1 se muestran las condiciones de presión (altura de columna de agua) con las que se logró cada dilución o punto de referencia de las curvas, la velocidad de flujo de ambos gases y el flujo total de la mezcla, así como la concentración teórica de etileno correspondiente.

En cada repetición se asoció la concentración teórica de etileno obtenida con el área bajo la curva del pico de etileno promedio de los cromatogramas generados. Los datos se sometieron a regresión lineal y se determinaron los parámetros de dispersión con el software de SigmaPlot $12 \circledR$ (Systat Software Inc., 2010).

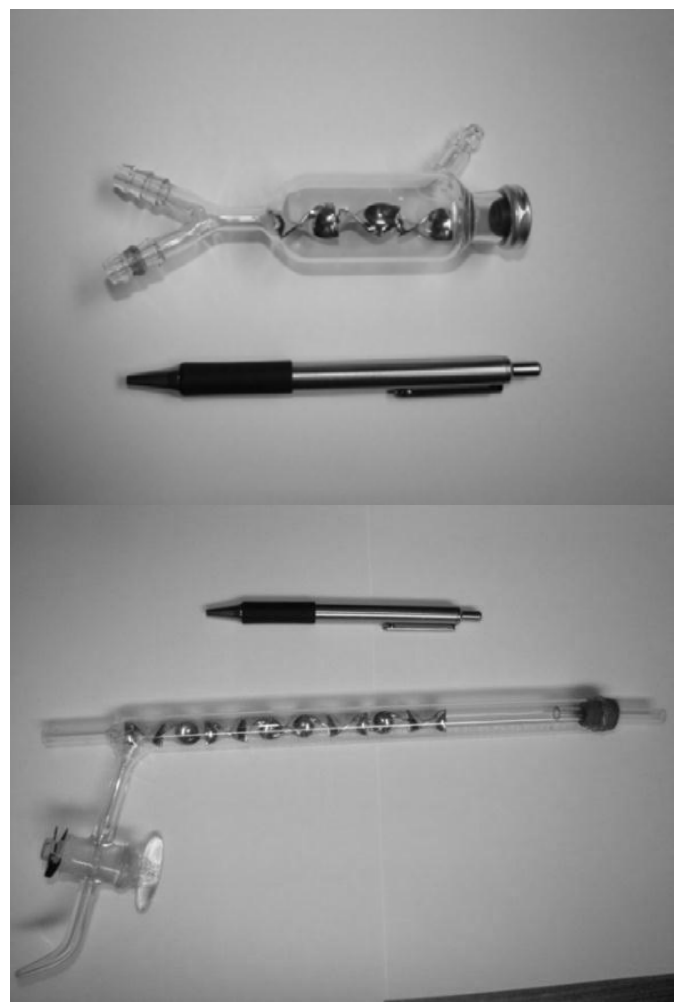

Figura 2. Detalles del sistema de mezcladores estáticos Kenics ${ }^{\circledR}$ (Chemineer, Inc.) empleados para mejorar la mezcla de gases.

Cuadro 1. Condiciones de presión (altura de la columna de agua), velocidades de flujo de etileno y nitrógeno y concentración teórica de etileno para cada punto de referencia de la curva de calibración.

\begin{tabular}{|c|c|c|c|c|c|}
\hline \multicolumn{3}{|c|}{ Etileno $\left(\mathrm{C}_{2} \mathrm{H}_{4}\right)$ Tablero 1} & \multicolumn{3}{|c|}{ Nitrógeno $\left(\mathrm{N}_{2}\right)$ Tablero 2} \\
\hline $\begin{array}{l}\text { Altura de columna } \\
\text { de agua }(\mathrm{cm})\end{array}$ & $\begin{array}{l}\text { Flujo de etileno } \\
\left(\mathrm{L} \mathrm{h}^{-1}\right)\end{array}$ & $\begin{array}{l}{\left[\mathrm{C}_{2} \mathrm{H}_{4}\right] \text { teórica }} \\
\left(\mathrm{mg} \mathrm{L}^{-1}\right)\end{array}$ & $\begin{array}{l}\text { Altura de la columna } \\
\text { de agua }(\mathrm{cm})\end{array}$ & $\begin{array}{l}\text { Flujo de } \mathrm{N}_{2} \\
\left(\mathrm{~L} \mathrm{~h}^{-1}\right)\end{array}$ & $\begin{array}{l}\text { Flujo total } \\
\left(\mathrm{L} \mathrm{h}^{-1}\right)\end{array}$ \\
\hline 0.0 & 0.0 & 0.0 & 24.5 & 11.04 & 11.04 \\
\hline 5.0 & 2.26 & 20.52 & 18.75 & 8.75 & 11.01 \\
\hline 10.5 & 4.66 & 42.36 & 13.25 & 6.34 & 11.00 \\
\hline 16.0 & 6.89 & 62.69 & 8.25 & 4.10 & 10.99 \\
\hline 21.5 & 9.02 & 81.92 & 4.0 & 1.99 & 11.01 \\
\hline 27.0 & 11.0 & 100 & 0.0 & 0.0 & 11.00 \\
\hline
\end{tabular}




\section{RESULTADOS Y DISCUSIÓN}

Al considerar la concentración de etileno como la variable independiente y el área promedio bajo la curva del pico como la variable dependiente, se generaron las ecuaciones 2 y 3 . Éstas ecuaciones representan el comportamiento del área bajo la curva del cromatograma generado por la concentración de etileno, sin utilizar mezcladores estáticos (ASM) y cuando éstos se emplean (ACM). En la práctica se evalúa el área bajo la curva del pico cromatográfico generado por una concentración desconocida del gas de interés (etileno, en este caso), por lo cual es necesario despejar de la ecuación la concentración del etileno $([E])$ :

$A_{S M}=26.0648[E]+0.1640[E]^{2}$

$A_{C M}=26.8043[E]+0.1366[E]^{2}$

Estas ecuaciones carecen de ordenada al origen, lo que significa que si la concentración de etileno fuera cero el área bajo la curva también sería cero. Este hecho corresponde a lo observado experimentalmente. Los coeficientes de regresión mostraron valores altos en ambos casos; sin embargo, cuando se empleó el sistema de mezcladores estáticos la $\mathrm{R}^{2}$ fue mayor (0.999) en comparación a la obtenida sin el uso de dicho sistema (0.986). Adicionalmente, cuando se empleó el sistema de mezcladores estáticos se observó una reducción en el error estándar para cada punto de referencia. Esta reducción osciló desde $53.2 \%$ hasta $87.4 \%$ (Cuadro 2). Estos resultados ponen en evidencia la mejora de la eficiencia del proceso de mezclado de gases con el uso del sistema de mezcladores estáticos, atribuible a los cambios de dirección de los flujos gaseosos en los mezcladores que incrementaron la turbulencia y favorecieron los procesos de transferencia de masa en el seno del fluido gaseoso, para dar como resultado una mezcla de gases más homogénea.

Las curvas de calibración de etileno diluido con nitrógeno generadas con y sin el uso del sistema de mezcladores estáticos dieron como resultado parámetros de regresión ligeramente diferentes (Ecuaciones 2 y 3 ). Si bien esto indica que se podrían tener diferencias pequeñas entre las estimaciones de los dos modelos, la confiabilidad de dicha predicción es mayor con el uso del sistema de mezcladores estáticos porque éstos reducen el error estándar. De hecho, cuando se calculan los intervalos de confianza de dichos modelos, como se muestra en la Figura 3, es evidente que la incertidumbre de la predicción es menor cuando se emplea el sistema con mezcladores estáticos. Es decir, el empleo de curvas de calibración construidas a partir de mezclas de etileno/nitrógeno elaboradas con el sistema con mezcladores estáticos permite predecir con menor incertidumbre las concentraciones de los gases en cuestión. El empleo de este sistema podrá contribuir en la generación de mejores datos experimentales de trabajos futuros, así como al ahorro de recursos y de tiempo.

Cuadro 2. Área bajo la curva del pico cromatográfico (promedio de tres lecturas) obtenida por cromatografía de gases para diferentes concentraciones $\left(\mathrm{mg} \mathrm{L}^{-1}\right)$ de etileno diluido con nitrógeno de alta pureza, error estándar y reducción (\%) del error estándar de cada dilución, en curvas obtenidas con (CM) o sin (SM) el uso de mezcladores estáticos.

\begin{tabular}{|c|c|c|c|c|c|}
\hline \multirow[b]{2}{*}{ Etileno $\left(\mathrm{mg} \mathrm{L}^{-1}\right)$} & \multicolumn{2}{|c|}{$\begin{array}{c}\text { Área bajo } \\
\text { la curva del pico }\end{array}$} & \multicolumn{2}{|c|}{$\begin{array}{c}\text { Error } \\
\text { estándar }\end{array}$} & \multirow[t]{2}{*}{$\begin{array}{l}\text { Reducción del } \\
\text { error estándar (\%) }\end{array}$} \\
\hline & SM & $\mathrm{CM}$ & $\mathrm{SM}$ & $\mathrm{CM}$ & \\
\hline 0.0 & 0 & 0 & 0 & 0 & 0 \\
\hline 20.62 & 739.6 & 627.3 & 64.5 & 14.3 & 77.7 \\
\hline 42.38 & 1486.3 & 1362.6 & 82.4 & 25.3 & 69.2 \\
\hline 62.69 & 2091.3 & 2234.3 & 115.6 & 14.6 & 87.36 \\
\hline 88.01 & 3246.0 & 3101.6 & 75.83 & 22.0 & 70.93 \\
\hline 100.00 & 4295.0 & 4052.0 & 157.9 & 73.8 & 53.24 \\
\hline
\end{tabular}



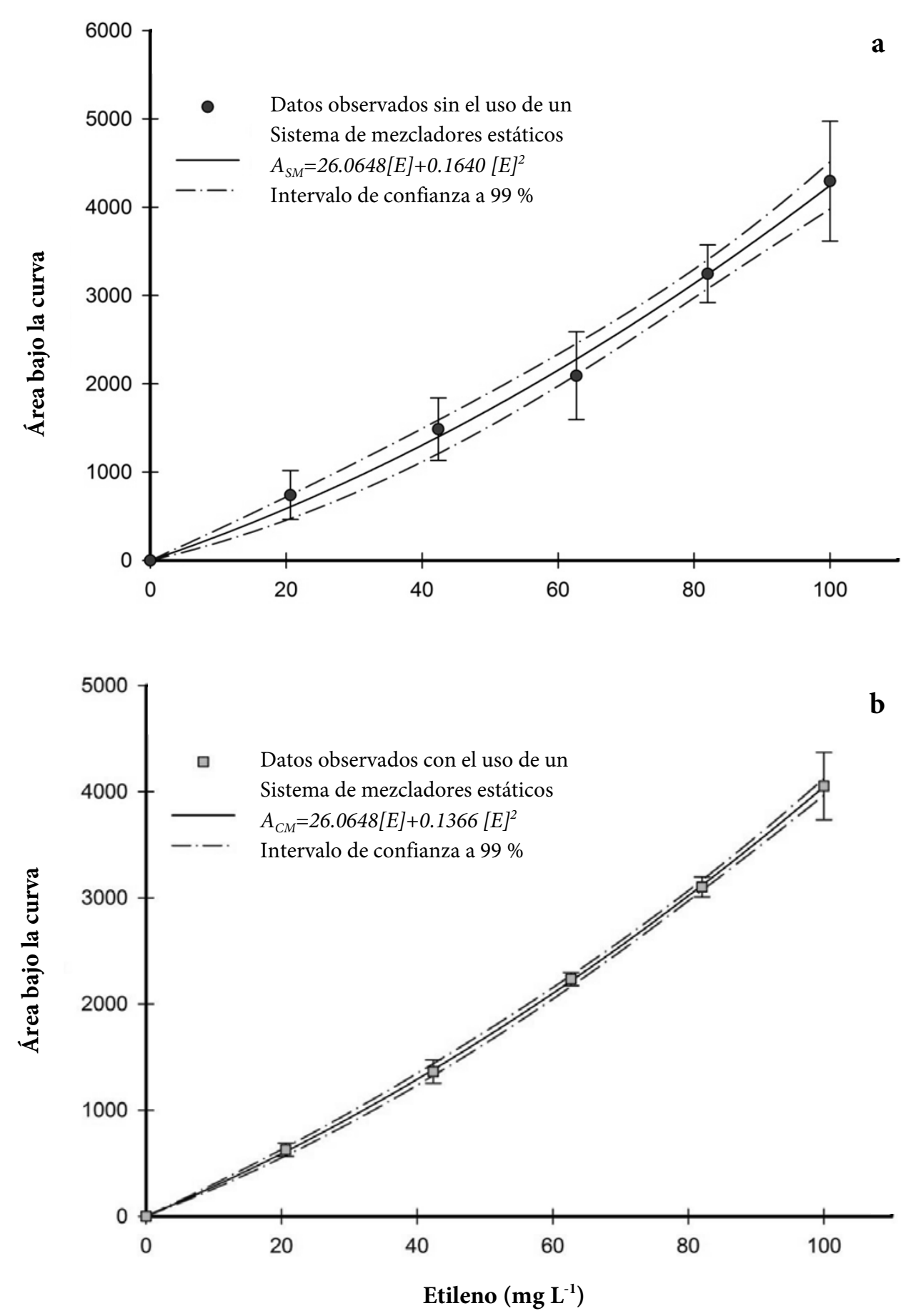

Figura 3. Curvas de calibración para diluciones de etileno con nitrógeno obtenidas sin (a) y con (b) el uso de mezcladores estáticos. Cada punto representa la media de tres repeticiones y las barras verticales indican la desviación estándar. 


\section{CONCLUSIONES}

El uso del sistema con mezcladores estáticos mejoró la precisión y confiabilidad de curvas de calibración de mezclas de etileno-nitrógeno para estudios de fisiología y tecnología postcosecha, al reducir el error estándar hasta en $87 \%$ en sus puntos de referencia. De esta forma se puede mejorar la precisión de las determinaciones de las concentraciones de dichos gases en este tipo de estudios. Los resultados del presente trabajo hacen un importante aporte metodológico en la preparación de mezclas de gases en el campo de la fisiología y tecnología postcosecha, particularmente en el área de atmósferas controladas o modificadas.

\section{BIBLIOGRAFÍA}

Barrué H, A Karoui, N Le Sauze, J Costes, F Illy (2001) Comparison of aerodynamics and mixing mechanisms of three mixers: Oxynator $^{\text {tw }}$ gas-gas mixer, KMA and SMI static mixers. Chem. Eng. J. 84:343-354.

Couvert A, C Sanchez, I Charron, A Laplanche, C Renner (2006) Static mixers with a gas continuous phase. Chem. Eng. Sci. 61:34293434.

Fourcade E, H C J Hoefsloot, G van Vliet, W Bunge, S M P Mutsers, P D Iedema (2001) The influence of micromixing on molecular weight distribution during controlled polypropylene degradation in a static mixer reactor. Chem. Eng. Sci. 56:65896603.

Fradette L, H Z Li, L Choplin, P Tanguy (2006) Gas/liquid dispersions with a SMXstatic mixer in the laminar regime. Chem. Eng. Sci. 61:3506-3518

Kader A A (2007a) Biología y tecnología postcosecha: un panorama. In: Tecnología Postcosecha de Cultivos Hortofrutícolas. A A Kader (ed). Universidad de California. Centro de Información e Investigación en Tecnología Postcosecha, División de Agricultura y Recursos Naturales. Serie de Horticultura Postcosecha 24. Trad. por ANR. Davis, California, USA. pp:4554.

Kader A A (2007b) Métodos de mezclado, muestreo y análisis de gases. In: Tecnología Postcosecha de Cultivos Hortofrutícolas. A A Kader (ed). Universidad de California. Centro de Información e Investigación en Tecnología Postcosecha. División de Agricultura y Recursos Naturales. Serie de Horticultura Postcosecha 24. Trad. por ANR. Davis, California, USA. pp:169174.

Patkar V C, A W Patwardhan (2011) Effect of jet angle and orifice shape in gas-gas mixer using CFD. Chem. Eng. Res. Design 89:904-920.

Pratt H K, D B Mendoza Jr (1979) Colorimetric determination of carbon dioxide for respiration studies. HortScience 14:175-176.

Systat Software, Inc. (2010) SigmaPlot 12 User's Guide. USA. Systat Software, Inc.

Tajima H, Y Yoshida, S Abiko, K Yamagiwa (2010) Size adjustment of spherical temperature-sensitive hydrogel beads by liquid-liquid dispersion using a Kenics static mixer. Chem. Eng. J. 156:479486. 